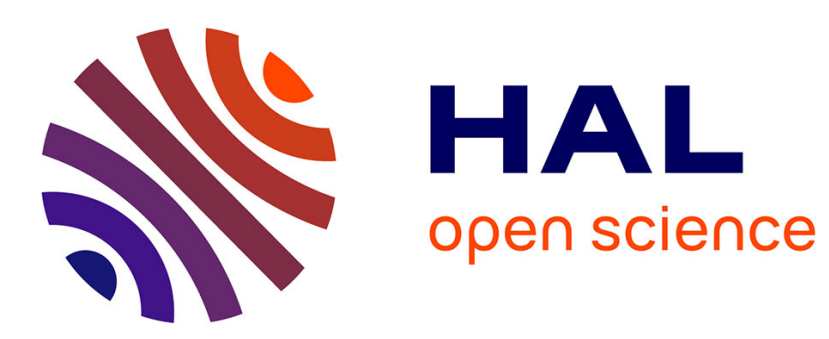

\title{
TECHNIQUES DE RENORMALISATION DE LA THÉORIE DES CHAMPS ET DYNAMIQUE DES PHÉNOMÈNES CRITIQUES
}

Cirano de Dominicis

\section{- To cite this version:}

Cirano de Dominicis. TECHNIQUES DE RENORMALISATION DE LA THÉORIE DES CHAMPS ET DYNAMIQUE DES PHÉNOMÈNES CRITIQUES. Journal de Physique Colloques, 1976, 37 (C1), pp.C1-247-C1-253. 10.1051/jphyscol:1976138 • jpa-00216466

\section{HAL Id: jpa-00216466 https://hal.science/jpa-00216466}

Submitted on 1 Jan 1976

HAL is a multi-disciplinary open access archive for the deposit and dissemination of scientific research documents, whether they are published or not. The documents may come from teaching and research institutions in France or abroad, or from public or private research centers.
L'archive ouverte pluridisciplinaire HAL, est destinée au dépôt et à la diffusion de documents scientifiques de niveau recherche, publiés ou non, émanant des établissements d'enseignement et de recherche français ou étrangers, des laboratoires publics ou privés. 


\title{
TECHNIQUES DE RENORMALISATION DE LA THÉORIE DES CHAMPS ET DYNAMIQUE DES PHÉNOMẼNES CRITIQUES
}

\author{
C. DE DOMINICIS
}

Service de Physique Théorique, CEN, Saclay, BP no 2, 91190 Gif-sur-Yvette, France

\begin{abstract}
Résumé. - La dynamique des phénomènes critiques telle qu'elle est décrite par les équations stochastiques de type Ginzburg-Landau dépendant du temps, avec ou sans loi de conservation, est étudiée par les techniques de renormalisation de la théorie des champs.

Le cas des systèmes comportant un couplage mode-mode est brièvement abordé.

Abstract. - The dynamics of critical phenomena as is described by stochastic equations of the Landau-Ginzburg type with or without conservation law, is studied by the technique of field renormalization.

The case of mode coupling systems is briefly touched upon.
\end{abstract}

1. Introduction. - Dans ce bref exposé nous nous bornons à présenter quelques résultats concernant la dynamique des phénomènes critiques telle qu'elle est décrite par les modèles stochastiques de Halperin, Hohenberg et $\mathrm{Ma}$ [1] (modèles étendus au cas du couplage mode-mode par Halperin, Hohenberg, Siggia [2] et Ma, Mazenko [3]). En particulier nous n'abordons pas le problème de l'établissement de ces équations. Les auteurs précédents utilisent la méthode itérative inventée par Wilson [4]. Ici, nous illustrons brièvement comment les méthodes de la théorie de la renormalisation des champs, déjà utilisées par Brézin, Le Guillou, Zinn-Justin [5] pour la statique des systèmes critiques, peuvent être aussi étendues et utilisées pour étudier les propriétés dynamiques. Cette méthode permet d'établir de façon claire et rigoureuse les lois d'échelles dynamiques, lorsqu'elles existent, lois d'échelles qui avaient été proposées [6,7] sur une base heuristique il y a déjà plusieurs années. En outre le calcul de l'exposant critique dynamique ainsi que des principaux exposants correctifs devient particulièrement compact $[8,9]$ permettant de retrouver et d'étendre les résultats de Halperin, Hohenberg, Ma.

Après avoir rappelé la méthode de Brézin, Le Guillou, Zinn-Justin, nous énoncerons quelques résultats concernant les systèmes dits de Ginzburg-Landau dépendant du temps (GLDT) sans loi de conservation, puis avec conservation de la variable énergie. Nous terminerons par quelques remarques concernant les systèmes avec couplage mode-mode.

2. Système GLDT sans loi de conservation. 2.1 Statique [5]. - Le Hamiltonien $\left(^{1}\right.$ ) qui gou-

(1) Nous utilisons tout au long de ce résumé le terme Hamiltonien pour désigner la quantité qui apparaît dans le poids statistique $\exp \{-\mathfrak{H}\}$, s'introduisant dans le calcul de fonctions de corrélations indépendantes du temps. Pour les quantités dépendant du temps, le poids est $\exp \{+\mathfrak{L}\}$, et $\mathfrak{L}$ est un Lagrangien. verne les propriétés statiques dans la région critique s'écrit [4]

$$
\begin{aligned}
\mathscr{H}\{\varphi\}=\frac{1}{2} \sum_{k}\left(r_{0}+k^{2}\right) & \varphi_{k} \cdot \varphi_{-k}+ \\
& +\frac{g_{0}}{4 !} \int \mathrm{d}^{d} x(\varphi(x) \cdot \varphi(x))^{2}
\end{aligned}
$$

où $\left({ }^{2}\right) \varphi(x)$ est le champ paramètre d'ordre (vecteur à $N$ composantes), $d=4-\varepsilon$ est la dimensionalité de l'espace, $g_{0}=u_{0} \Lambda^{\varepsilon}$ est la constante de couplage qui a la dimension $\Lambda^{\varepsilon}$ où $\Lambda$ est un vecteur de cut-off (de l'ordre de l'inverse de la maille du réseau si la forme continue (1) dérive d'une interaction sur un réseau) enfin

$$
r_{0}=r_{0 \mathrm{c}}+t
$$

où $t$ est une mesure linéaire de la température $(t=0$ à la température critique). Le domaine critique est caractérisé par $k \ll \Lambda$ et $t \ll \Lambda^{2}$. Les fonctions de corrélation sont calculées avec le poids $\exp \{-\mathcal{H}\}$ et la théorie de la renormalisation nous renseigne sur le comportement des fonctions de corrélations pour $k / \Lambda$ et $t / \Lambda^{2}$ tendant vers zéro. Ainsi la fonction de corrélation à deux points

$$
<\varphi_{k} \varphi_{-k}>\equiv \chi(k)
$$

s'écrit, dans le domaine critique

$$
\chi\left(k ; u_{0}, t, \Lambda\right)=Z\left(u_{0} ; \frac{t}{\Lambda^{2}}\right) \chi_{\mathrm{R}}\left(k ; u_{\mathrm{R}}, t_{\mathrm{R}}\right)
$$

où dans la fonction de corrélation renormalisée $\chi_{R}$ la dépendance en le cut-off $\Lambda$ n'apparaît que via $u_{R}$ constante de couplage renormalisée et $t_{\mathrm{R}} \equiv \xi^{-2}$ masse renormalisée (inverse du carré de la longueur de corrélation $\xi$ ).

(2) On utilise $\varphi(x)$ et $\varphi_{k}$ pour le paramètre d'ordre et son transformé de Fourier, de même plus loin $\varphi(x, t)$, et $\varphi_{t \omega}$. 
La forme (3) manifeste l'existence de lois d'échelles par le fait que l'unité de longueur $\Lambda^{-1}$ a explicitement disparu dans $\chi_{\mathrm{R}}$. Exprimant cette propriété (en faisant varier $\Lambda$ à $u_{\mathrm{R}}, t_{\mathrm{R}}$ fixés) on obtient l'équation du groupe de renormalisation (sous sa forme différentielle)

$$
\begin{aligned}
& \left\{\Lambda \frac{\partial}{\partial \Lambda}+W\left(u_{0}, \frac{t}{\Lambda^{2}}\right) \frac{\partial}{\partial u_{0}}-\gamma\left(u_{0}, \frac{t}{\Lambda^{2}}\right)-\right. \\
& \left.\quad-\left(v^{-1}\left(u_{0}, \frac{t}{\Lambda^{2}}\right)-2\right) t \frac{\partial}{\partial t}\right\} \chi^{-1}\left(k ; u_{0}, t, \Lambda\right)=0
\end{aligned}
$$

avec les définitions

$$
\begin{aligned}
W\left(u_{0}, \frac{t}{\Lambda^{2}}\right) & =\left.\Lambda \frac{\partial}{\partial \Lambda} u_{0}\right|_{\mathrm{R}} \\
\gamma\left(u_{0}, \frac{t}{\Lambda^{2}}\right) & =-\left.\Lambda \frac{\partial}{\partial \Lambda} \ln Z\right|_{\mathrm{R}} \\
\nu^{-1}\left(u_{0}, \frac{t}{\Lambda^{2}}\right)-2 & =-\left.\Lambda \frac{\partial}{\partial \Lambda} \ln t\right|_{\mathrm{R}} .
\end{aligned}
$$

En outre puisque $W, \gamma$ et $v^{-1}$ ont des limites finies $\left({ }^{3}\right)$ pour $t / \Lambda^{2} \rightarrow 0$, les fonctions ne dépendent que de $u_{0}$ et peuvent être construites comme des développements en puissances de $u_{0}$ et $\varepsilon$, par exemple

$$
W\left(u_{0}\right)=-\varepsilon u_{0}+\frac{N+8}{6} u_{0}^{2}+O\left(u_{0}^{3}, u_{0}^{2} \varepsilon\right) .
$$

L'équation du groupe de renormalisation (G. R.) (4) conduit (par la méthode des caractéristiques) à relier la fonction de corrélation inverse $\chi^{-1}\left(k ; u_{0}, t, \Lambda\right)$ à la même fonction évaluée pour des valeurs d'une constante de couplage effective $u_{0}(\rho)$ et d'une température effective $t(\rho), \rho$ étant un paramètre arbitraire de dilatation des longueurs. Plus précisément :

- Si $t=0$ (c'est-à-dire à la température critique) :

$$
\begin{aligned}
& \chi^{-1}\left(k ; u_{0}, \Lambda\right)=\rho^{2} \exp \left\{-\int_{1}^{\rho} \frac{\mathrm{d} \rho^{\prime}}{\rho^{\prime}} \eta\left[u_{0}\left(\rho^{\prime}\right)\right]\right\} \times \\
& \times \chi^{-1}\left(\frac{k}{\rho} ; u_{0}(\rho), \Lambda\right)
\end{aligned}
$$

la fonction $u_{0}(\rho)$ définie par

$$
\rho \frac{\mathrm{d} u_{0}}{\mathrm{~d} \rho}(\rho)=W\left[u_{0}(\rho)\right], \quad u_{0}(1)=u_{0} .
$$

Si on choisit $\rho=k / \Lambda \ll 1$ (domaine critique)

$$
u_{0}(\rho)=u_{0}^{*}+c \rho^{\omega}+\cdots
$$

(3) En effet $W, \gamma, v^{-1}$, peuvent s'exprimer en termes des fonctions de corrélations évaluées à des valeurs arbitraires mais fixes des vecteurs d'ondes, valeurs qui peuvent être choisies de façon à les rendre essentiellement indépendantes de $t$. où $u_{0}^{*}$ est un point fixe stable infrarouge si l'exposant $\omega>0$

$$
\left.\begin{array}{l}
u_{0}^{*}=\frac{6}{N+8}+\cdots \\
\omega=W^{\prime}\left(u_{0}^{*}\right)=\varepsilon+\cdots
\end{array}\right\}
$$

Le comportement de la fonction de corrélation dans le domaine critique est alors gouverné par la valeur de point fixe de $u_{0}(\rho)$ soit $u_{0}^{*}$. Plus précisément

$$
\begin{aligned}
\chi^{-1}\left(k ; u_{0}, \Lambda\right) \simeq\left(\frac{k}{\Lambda}\right)^{2-\eta^{*}} & \chi^{-1}\left(\Lambda ; u_{0}^{*}, \Lambda\right) \times \\
& \times\left[1+C\left(\frac{k}{\Lambda}\right)^{\omega}+\cdots\right]
\end{aligned}
$$

c'est-à-dire qu'on obtient à la fois l'exposant critique $\eta^{*} \equiv \eta\left(u_{0}^{*}\right)$ et les corrections principales, données par l'exposant $\omega$.

- Si $t \neq 0:$ la solution est de même forme mais dans le membre de droite de (7) $\chi^{-1}$ devient

$$
\chi^{-1}\left(\frac{k}{\rho} ; u_{0}(\rho), \frac{t(\rho)}{\rho^{2}}, \Lambda\right)
$$

avec

$$
\rho \frac{\mathrm{d}}{\mathrm{d} \rho} \ln t(\rho)=2-v^{-1}\left[u_{0}(\rho)\right], \quad t(1)=t
$$

soit pour $\rho=k / \Lambda \ll 1$,

$$
t(\rho) \sim t \rho^{2-v^{-1}\left(u_{o}^{*}\right)}+\cdots .
$$

Au lieu de (11) on a alors,

$$
\begin{aligned}
\chi^{-1}\left(k ; u_{0}, t, \Lambda\right) & \simeq\left(\frac{k}{\Lambda}\right)^{2-\eta^{*}} \times \\
& \times \chi^{-1}\left(\Lambda ; u_{0}^{*}, \frac{t}{\Lambda^{2}}\left(\frac{k}{\Lambda}\right)^{v^{*-1}}, \Lambda\right)+\cdots
\end{aligned}
$$

c'est-à-dire une forme exhibant la loi d'échelle

$$
\chi^{-1}(k) \sim k^{2-\eta^{*}} f(k \xi)
$$

avec $\xi \sim t^{-v^{*}}$, les termes correctifs étant obtenus comme précédemment par développement de Taylor de $\chi^{-1}$ autour des valeurs principales.

2.2 Dynamique [8]. - La dynamique, dans la région critique, peut être décrite par une équation stochastique de type Langevin-Ginzburg-Landau

$$
\frac{\partial \varphi_{k}(t)}{\partial t}=-\Gamma_{0}\left\{\frac{\delta \mathscr{H}_{0}\{\varphi(t)}{\delta \varphi_{k}(t)} \underline{y}+h_{k}(t)\right\}+\eta_{k}(t)
$$

où la fonctionnelle $\mathfrak{H}$ est donnée par (1). Ici, $h_{k}(t)$ est un champ extérieur, $\Gamma_{0}$ l'inverse d'un temps de relaxation caractérisant les grands vecteurs d'onde et 
$\eta_{k}(t)$ le champ aléatoire gouverné par une loi de probabilité qu'on peut prendre gaussienne avec

$$
\begin{aligned}
\left\langle\eta_{k}(t)>\right. & =0 \\
\left\langle\eta_{k}(t) \eta_{k^{\prime}}\left(t^{\prime}\right)>\right. & =2 \Gamma_{0} \delta_{k k^{\prime}} \delta\left(t-t^{\prime}\right) .
\end{aligned}
$$

L'éq. (17) traduit le fait que $\eta_{k}(t)$ représente la partie fluctuant rapidement dans le temps (temps caractéristique très court). Cette équation de Langevin-Ginzburg-Landau peut être obtenue par exemple à partir du modèle d'Ising auquel on adjoint une loi de probabilité pour le renversement d'un spin (modèle de Glauber [10]). On peut montrer [11] que pour ce qui est du calcul des fonctions de corrélations (réponses linéaires) l'éq. (16) peut être remplacée par le Lagrangien

$$
\begin{aligned}
-\mathfrak{L}\left\{\varphi_{k \omega}\right\}=- & \frac{1}{2} \sum_{k \omega} \frac{i \omega}{\Gamma_{0}} \varphi_{k \omega} \varphi_{-k-\omega}+ \\
& +\mathscr{H}\left\{\varphi_{k \omega}\right\}+\sum_{k \omega} h_{k \omega} \varphi_{-k,-\omega}
\end{aligned}
$$

où les valeurs moyennes $\left\langle\varphi_{k \omega} \varphi_{-k,-\omega}\right\rangle \equiv \chi(k, \omega)$ sont calculées avec le poids $\exp \{+\mathcal{L}\}$.

La dépendance en $\Lambda$ apparaît, via la procédure de renormalisation, par l'intermédiaire des quantités renormalisées introduites ci-dessus $\left(t_{\mathrm{R}}, Z, u_{\mathrm{R}}\right)$ pour la partie statique $\chi(k)=\chi(k, \omega=0)$ de la réponse linéaire. Cette partie statique donne bien, comme le

montre un simple comptage de puissances, une fonction à deux points divergeant comme $\Lambda^{2}$ (renormalisation de masse $t_{R}$, et de fonction d'onde $Z$ ) et une fonction à quatre points divergeant comme $\ln A$ (renormalisation de constante de couplage $u_{\mathrm{R}}$ ). La partie dynamique $\chi(k, \omega)-\chi(k)$ s'annule comme $\omega$ et possède donc un degré de divergence inférieur de deux degrés ( $\omega$ étant homogène à $k^{2}$ ) ce qui ne laisse pour la partie dynamique qu'une divergence en $\ln A$ pour la fonction à deux points. Cette divergence nouvelle donne lieu à une renormalisation supplémentaire $\Gamma_{0} \rightarrow \Gamma_{\mathrm{R}}$, conduisant à la forme (qui peut être établie en toute rigueur)

$$
\chi\left(k, \frac{i \omega}{\Gamma_{0}} ; u_{0}, t, \Lambda\right)=Z\left(u_{0}\right) \chi_{\mathrm{R}}\left(k, \frac{i \omega}{\Gamma_{\mathrm{R}}} ; u_{\mathrm{R}}, t_{\mathrm{R}}\right) .
$$

Si on varie $A$ à $t_{\mathrm{R}}, u_{\mathrm{R}}, \Gamma_{\mathrm{R}}$ fixés on obtient l'équation du $\mathrm{G}$. R. étendue au cas dynamique

$$
\left\{\mathfrak{D}_{\mathrm{st}}-\left[z\left(u_{0}\right)-2\right] \omega \frac{\partial}{\partial \omega}\right\} \chi^{-1}\left(k, \frac{i \omega}{\Gamma_{0}} ; u_{0}, t, \Lambda\right)=0
$$

où $\mathfrak{D}_{\mathrm{st}}$ est l'opérateur différentiel de l'équation du G. R. statique (4) et la fonction $z\left(u_{0}\right)$ est définie par

$$
z\left(u_{0}\right)-2=\left.\Lambda \frac{\partial}{\partial \Lambda} \ln \Gamma_{\mathrm{o}}\right|_{\mathrm{R}}
$$

Comme en (7) la solution de (20) s'écrit

avec

$$
\chi^{-1}\left(k, \frac{i \omega}{\Gamma_{0}} ; u_{0}, t, A\right)=\rho^{2} \exp \left\{-\int^{\rho} \frac{\mathrm{d} \rho^{\prime}}{\rho^{\prime}} \eta\left[u_{0}\left(\rho^{\prime}\right)\right]\right\} \chi^{-1}\left(\frac{k}{\rho}, \frac{i \omega}{\rho^{2} \Gamma_{0}(\rho)} ; u_{0}(\rho), \frac{t(\rho)}{\rho^{2}}, \Lambda\right)
$$

$$
\rho \frac{\mathrm{d}}{\mathrm{d} \rho} \ln \left[\rho^{2} \Gamma_{0}(\rho)\right]=z\left[u_{0}(\rho)\right]
$$

et $u_{0}(\rho), t(\rho)$ donnés par $(8),(12)$. Dans le domaine critique $(\rho=k / \Lambda \ll 1)$ on obtient

$$
\chi^{-1}\left(k, \frac{i \omega}{\Gamma_{0}} ; u_{0}, t, \Lambda\right) \simeq k^{2-\eta^{*}} \chi^{-1}\left(\Lambda, \frac{i \omega}{\Gamma_{0}} \frac{A^{z^{*}}}{k} ; u_{0}^{*}, t\left(\frac{\Lambda}{k}\right)^{1 / v^{*}}, \Lambda\right)
$$

de la forme anticipée par l'hypothèse des lois d'échelle dynamique [6,7]

$$
\chi^{-1}\left(k, \frac{i \omega}{\Gamma_{0}}\right) \simeq k^{2-\eta^{*}} g\left(\frac{i \omega}{k^{z^{*}}}, k \xi\right),
$$

c'est-à-dire faisant apparaître une fréquence caractéristique (présentant un rétrécissement critique),

où

$$
\omega_{\mathrm{c}}(k, t) \simeq k^{z^{*}} h(k \xi)
$$

$$
z^{*}=z\left(u_{0}^{*}\right) \text {. }
$$

Les corrélations obtenues en développement de Taylor $u_{0}(\rho), t(\rho), \rho^{2} \Gamma_{0}(\rho)$ autour de leurs valeurs asymptotiques $u_{0}^{*}, t \rho^{-1 / v^{*}}, \Gamma_{0} \rho^{Z^{*}}$ sont à nouveau gouvernées par l'exposant $\omega=W^{\prime}\left(u_{0}^{*}\right)$.

Les calculs peuvent être poussés sans difficultés pour $z\left(u_{0}\right)$ en puissances de $u_{0}$ et $\varepsilon$, donnant pour l'exposant critique dynamique, à l'ordre $\varepsilon^{3}$,

$$
z^{*}=2+\frac{\varepsilon^{2}}{2}\left(6 \ln \frac{4}{3}-1\right) \frac{N+2}{(N+8)^{2}}\left[1+\varepsilon\left(\frac{6(3 N+14)}{(N+8)^{2}}-3,162\right)\right] .
$$

La comparaison avec les calculs de H. Yahata [12] obtenus à partir de développements de haute température n'est pas probante (les barres d'erreur sur $z^{*}$ sont données par les erreurs relatives sur $\eta^{*}$ ). 
3. Modèle GLDT avec loi de conservation. - Lorsque certaines variables du système obéissent à une loi de conservation (ici l'énergie) ces variables relaxent vers l'équilibre suivant un mode lent (mode hydrodynamique). Le mode critique étudié est aussi un mode lent (rétrécissement critique qui apparaît sur $\omega_{c}(k) \sim k^{z^{*}}, z^{*}>2$ ) qui a tout lieu d'être couplé aux modes conservés présents. On étudie donc un modèle où une variable énergie $E(x, t)$ se couple à la variable paramètre d'ordre $\varphi(x, t)$ (modèle qu'on peut réaliser comme une variante du modèle de Glauber [10]).

3.1 Statique. — On prend à cet effet

$$
\mathscr{H}\{\varphi, E\}=\mathscr{H}\{\varphi\}+\gamma_{0} \Lambda^{\varepsilon / 2} \int \mathrm{d}^{d} x E(x) \varphi^{2}(x)+\frac{1}{2 c_{0}} \int \mathrm{d}^{d} x E^{2}(x) .
$$

On sait $[4,5]$ que $E(x) \simeq \varphi^{2}(x)+$ Cte et donc (29) est équivalent à (1), ce qu'on vérifie en intégrant sur $E(x)$ le poids $\exp \{-\mathfrak{H}(\varphi, E)\}$. On obtient le poids réduit pour l'espace des seuls $\varphi(x), \exp \{-\widetilde{H}(\varphi)\}$ où

$$
\widetilde{\mathscr{H}}\{\varphi\}=\mathscr{H}_{0}\{\varphi\}+\frac{\tilde{u}_{0} A^{\varepsilon}}{4 !} \int \mathrm{d}^{d} x(\varphi(x) \cdot \varphi(x))^{2}
$$

avec

$$
\tilde{u}_{0}=u_{0}-12 \gamma_{0}^{2} c_{0}
$$

3.2 Dynamique [9]. - Outre l'équation stochastique (16) déjà écrite plus haut, où il faut naturellement remplacer $\mathscr{H}\{\varphi\}$ par $\mathscr{H}\{\varphi, E\}$,

$$
\frac{\partial}{\partial t} \varphi_{k}(t)=-\Gamma_{0}\left\{\frac{\delta \mathcal{H e}\{\varphi, E\}}{\delta \varphi_{k}(t)}+h_{k}(t)\right\}+\eta_{k}(t)
$$

la relaxation vers l'équilibre est maintenant gouvernée par une deuxième équation

$$
\frac{\partial}{\partial t} E_{k}(t)=-\Lambda_{0} k^{2}\left\{\frac{\delta \mathscr{H}\{\varphi, E\}}{\delta E_{k}(t)}+j_{k}(t)\right\}+\zeta_{k}(t) .
$$

La variable aléatoire $\zeta_{k}(t)$ est associée à une loi de probabilité gaussienne définie par

$$
\left.\begin{array}{rl}
<\zeta_{k}(t)> & =0 \\
<\zeta_{k}(t) \zeta_{k^{\prime}}\left(t^{\prime}\right)> & =2 k^{2} \Lambda_{0} \delta_{k k^{\prime}} \delta\left(t-t^{\prime}\right)
\end{array}\right\}
$$

On notera que

$$
\frac{\partial}{\partial t} E_{0}(t)=0
$$

exprime la loi de conservation (globale) de l'énergie.

A nouveau l'usage de (31) et (32) peut être remplacé [11] par le Lagrangien

$$
-\mathcal{L}\left\{\varphi_{k \omega}, E_{k \omega}\right\}=-\frac{1}{2} \sum_{k \omega}\left(\frac{i \omega}{\Gamma_{0}} \varphi_{k, \omega} \varphi_{-k,-\omega}+\frac{i \omega}{\Lambda_{0} k^{2}} E_{k, \omega} E_{-k,-\omega}\right)+\mathcal{H}\left\{\varphi_{k \omega}, E_{k \omega}\right\} .
$$

L'élimination, par intégration, des variables $E_{k \omega}$ conduit au Lagrangien effectif

$$
-\tilde{\mathcal{L}}\left\{\varphi_{k \omega}\right\}=-\mathcal{L}\left\{\varphi_{k \omega}, E=0\right\}-\frac{\gamma_{0}^{2}}{2} \sum_{k \omega}\left[\frac{-i \omega}{\Lambda_{0} k^{2}}+\frac{1}{c_{0}}\right]^{-1}\left(\sum_{k_{1} \omega_{1}} \varphi_{k+k_{1}, \omega+\omega_{1}} \varphi_{-k_{1},-\omega_{1}}\right)^{2}
$$

c'est-à-dire à un Lagrangien possédant un vertex singulier. Cette singularité reflète, partiellement, les difficultés rencontrées par l'approche microscopique où l'on essaie de construire un Lagrangien effectif en appliquant la méthode itérative de Wilson à un Lagrangien microscopique. Les modes conservés donnent alors lieu à des singularités dans les différents vertex effectifs. Les formes semi-phénoménologiques d'équations couplées du type (31), (32) simulent ces singularités en les remplaçant par l'adjonction explicite de variables conservées, convenablement couplées au champ du paramètre d'ordre. obtient

Ce système possède deux paramètres $\gamma_{0}$ et $\lambda_{0} \equiv \Lambda_{0} / \Gamma_{0}$ outre les précédents, $u_{0}, t$. Après renormalisation on

$$
\chi\left(k, \frac{i \omega}{\Gamma_{0}} ; u_{0}, \gamma_{0}, t, \lambda_{0}, A\right)=Z\left(u_{0}\right) \chi_{\mathrm{R}}\left(k, \frac{i \omega}{\Gamma_{\mathrm{R}}} ; u_{\mathrm{R}}, \gamma_{\mathrm{R}}, t_{\mathrm{R}}, \lambda_{\mathrm{R}}\right)
$$


Soit pour l'équation du G. R.

$$
\left\{\mathfrak{D}_{\mathrm{st}}-\left[z\left(u_{0}, \gamma_{0}, \lambda_{0}\right)-2\right] \omega \frac{\partial}{\partial \omega}+W_{\gamma_{0}}\left[u_{0}, \gamma_{0}\right] \frac{\partial}{\partial \gamma_{0}}+W_{\lambda_{0}}\left[u_{0}, \gamma_{0}, \lambda_{0}\right] \frac{\partial}{\partial \lambda_{0}}\right\} \chi^{-1}\left(k, \frac{i \omega}{\Gamma_{0}}\right)=0
$$

où

$$
W_{\gamma_{0}}=\left.\Lambda \frac{\partial \gamma_{0}}{\partial \Lambda}\right|_{\mathbf{R}}, \quad W_{\lambda_{0}}=\left.\Lambda \frac{\partial \lambda_{0}}{\partial \Lambda}\right|_{\mathbf{R}}
$$

sont les deux nouvelles fonctions de Wilson calculables par perturbation.

On peut montrer que ces fonctions ont les propriétés suivantes :

$$
W_{\gamma_{0}}=-\frac{\gamma_{0}}{2}\left[\eta_{\mathrm{E}}\left(\tilde{u}_{0}, \gamma_{0}\right)+2 \eta_{\varphi^{2}}\left(\tilde{u}_{0}\right)+\varepsilon\right]
$$

avec

$$
\left.\begin{array}{rl}
\eta_{\mathrm{E}}\left(\tilde{u}_{0}, \gamma_{0}\right) & =\left.\Lambda \frac{\partial}{\partial \Lambda} \ln Z_{\mathrm{E}}\right|_{\mathrm{R}}=-\gamma_{0}^{2} B\left(\tilde{u}_{0}\right) \\
\eta_{\varphi^{2}}\left(\tilde{u}_{0}\right) & =\left.\Lambda \frac{\partial}{\partial \Lambda} \ln Z_{\varphi^{2}}\right|_{\mathrm{R}}
\end{array}\right\}
$$

où $\eta_{\mathrm{E}}$ est à la fonction de corrélation énergie, ce que $\eta$ est à $\chi(k), Z_{\mathrm{E}}$ est la constante de renormalisation de fonction d'onde pour le champ $E, Z_{\varphi^{2}}$ pour les insertions $\varphi^{2}$. La fonction de Wilson $W_{\gamma_{0}}$ admet deux points fixes

$$
\begin{array}{ll}
\gamma_{0,1}^{*}=0 & W_{\gamma_{0}}^{\prime}\left(\gamma_{01}^{*}\right)=-(\alpha / v) \\
\gamma_{0,2}^{*}=-\left(\alpha / v B\left(\tilde{u}_{0}^{*}\right)\right) & W_{\gamma_{0}}^{\prime}\left(\gamma_{02}^{*}\right)=+(\alpha / v)
\end{array}
$$

$\tilde{u}_{0}^{*}$ étant la valeur du point fixe décrit plus haut (éq. (10)). De même, par définition du paramètre $\lambda_{0}$, on tire

$$
W_{\lambda_{0}}=-\lambda_{0}\left[z\left[\tilde{u}_{0}, \gamma_{0}, \lambda_{0}\right]-2+\eta_{\mathrm{E}}\left[\tilde{u}_{0}, \gamma_{0}\right]\right]
$$

avec

$$
\begin{aligned}
z\left[\tilde{u}_{0}, \gamma_{0}, \lambda_{0}\right] & =2+\frac{\gamma_{0}^{2}}{(1+\lambda)}\left[1-\frac{\varepsilon}{2}+\frac{\varepsilon}{2} \ln (1+\lambda)\right]+\tilde{u}_{0}^{2} \frac{N+2}{72}\left[6 \ln \frac{4}{3}-1\right] \\
& +\frac{\tilde{u}_{0} \gamma_{0}^{2}(N+2)}{6(1+\lambda)}\left[1+3 \ln \frac{4}{3}-\ln (1+\lambda)\right]+\frac{N \gamma_{0}^{4}}{4(1+\lambda)^{2}}\left[1+3 \ln \frac{4}{3}-\lambda-\ln (1+\lambda)\right] \\
& +\frac{\gamma_{0}^{4}}{2(1+\lambda)^{3}}\left[1-\lambda-\lambda^{2}+3(1+\lambda) \ln \frac{4}{3}+(2+\lambda)\left[2 \ln (1+\lambda)-\ln 2 \lambda-\ln \left(1+\frac{\lambda}{2}\right)\right]\right]+\ldots .
\end{aligned}
$$

Comme ci-dessus, la résolution de (37) introduit les paramètres effectifs $\tilde{u}_{0}(\rho), t(\rho), \Gamma_{0}(\rho)$ définis par $(8,12,23)$ où la fonction $z\left(\tilde{u}_{0}, \gamma_{0}, \lambda_{0}\right)$ est donnée par (43) et $\gamma_{0}(\rho), \lambda_{0}(\rho)$ définis par

$$
\begin{aligned}
& \rho \frac{\mathrm{d}}{\mathrm{d} \rho} \gamma_{0}(\rho)=W_{\gamma_{0}}\left[\tilde{u}_{0}(\rho), \gamma_{0}(\rho)\right] \\
& \rho \frac{\mathrm{d}}{\mathrm{d} \rho} \lambda_{0}(\rho)=W_{\lambda_{0}[}\left[\tilde{u}_{0}(\rho), \gamma_{0}(\rho), \lambda_{0}(\rho)\right] .
\end{aligned}
$$

Les zéros des fonctions $W_{u_{0}}, W_{\gamma_{0}}$ et $W_{\lambda_{0}}$ définissent les points fixes $u_{0}^{*}, \gamma_{0}^{*}, \lambda_{0}^{*}$, points fixes qui sont stables si les trois dérivées $W_{\tilde{u}_{0}}^{\prime}, W_{\gamma_{0}}^{\prime}, W_{\lambda_{0}}^{\prime}$ calculées aux points fixes sont positives. En ces points les exposants critiques sont donnés par $\eta^{*}, v^{*}$ (identiques au cas statique) et $z^{*}$, les corrections principales par $W_{\tilde{\varkappa}_{0}}^{\prime}, W_{\gamma_{0}}^{\prime}, W_{\lambda_{0}}^{\prime}$. 
Dans le plan $\varepsilon, N$ on compte quatre régions (Fig. 1):

$\begin{array}{lcccc} & \frac{\gamma_{0}^{*}}{2} & \frac{\lambda_{0}^{*}}{-} & \begin{array}{c}z^{*}-2 \\ \text { système }\end{array} & \begin{array}{c}\text { exposants correctifs } \\ \text { Ia }:(\alpha<0)\end{array} \\ \text { Ib }:(\alpha>0) & \neq 0 & +\infty & \begin{array}{c}\text { découplé } \\ \text { découplème }\end{array} & \omega,-\frac{\alpha}{v} \\ \text { II }:(\alpha>0) & \neq 0 & \text { fini } & \alpha / v & \omega, \frac{\alpha}{v} \\ \text { III }:(\alpha>0) & \neq 0 & 0 & & \begin{array}{c}\alpha, \frac{\alpha}{v},-\lambda^{*} \frac{\partial}{\partial \lambda^{*}} z\left(\lambda^{*}\right) \\ \text { pas de loi d'échelle }(?) \\ \omega_{\mathrm{c}}(k) \sim k^{z^{*}} \ln ^{2} k\end{array}\end{array}$

Toutefois le cas de la région III n'est pas complètement élucidé, le comportement en $k^{z^{*}} \ln ^{2} k$ n'étant que celui de l'ordre le plus bas en $\varepsilon$.

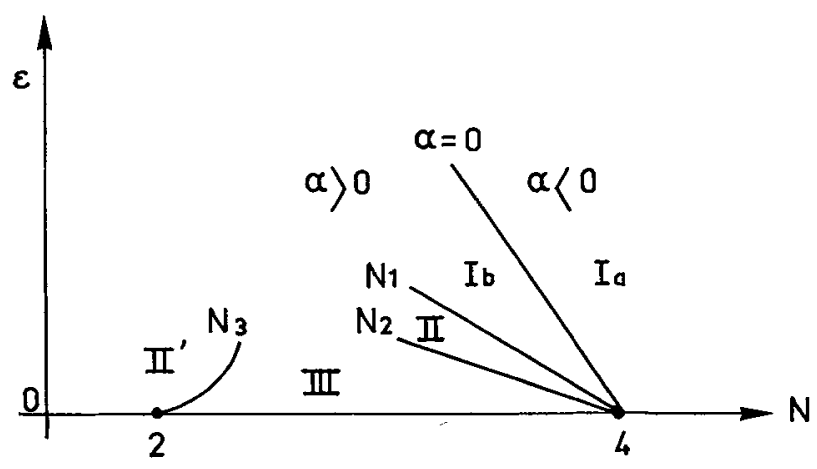

FIG. 1. - Topologie du plan $(\varepsilon, N)$ pour le système GLDT avec conservation de l'énergie. Les frontières des domaines sont définies par $\left(c=6 \ln \frac{4}{3}-1\right)$ :

$$
\begin{aligned}
& N_{1}=4-\varepsilon\left(4+\frac{c}{4}\right)+O\left(\varepsilon^{2}\right) \\
& N_{2}=4-\varepsilon\left(4+\frac{c}{2}\right)+O\left(\varepsilon^{2}\right) \\
& N_{3}=2+a \varepsilon|\ln \varepsilon|+\cdots
\end{aligned}
$$

3.3 COMPORTEMENT DES COEFFICIENTS CINÉTIQUeS OU DE TRANSPORT. - Dans les régions où il y a loi d'échelle, la démonstration esquissée ci-dessus conduit à des réponses linéaires $\chi_{\varphi \varphi}$ et $\chi_{\mathrm{EE}}$ pour les champs $\varphi$ et E de la forme

$$
\begin{aligned}
& \chi_{\varphi \varphi \varphi}^{-1}(k, \omega)=k^{2-\eta^{*}} f\left(\frac{\omega}{k^{z^{*}}}, k \xi\right) \\
& \chi_{E E}^{-1}(k, \omega)=k^{-\eta_{E}^{*}} g\left(\frac{\omega}{k^{z^{*}}}, k \xi\right)
\end{aligned}
$$

avec

$$
\eta_{\mathrm{E}}^{*}=\sup \left(0, \frac{\alpha}{v}\right) \equiv \frac{\tilde{\alpha}}{v}
$$

C'est-à-dire que dans les deux cas, la fréquence caractéristique s'écrit

$$
\omega_{\mathrm{c}}(k, t)=k^{z^{*}} h(k \xi) \text {. }
$$

On peut définir le coefficient cinétique $\Gamma(k)$ ou de transport (pour la quantité conservée) $\Lambda(k)$ par

$$
\begin{aligned}
\Gamma(k) & \equiv \omega_{\mathrm{c}}(k) \chi_{\varphi \varphi}(k) & \Gamma(k) & \sim k^{z^{*}-2+\eta^{*}} \\
k^{2} \Lambda(k) & \equiv \omega_{\mathrm{c}}(k) \chi_{\mathrm{EE}}(k) & \Lambda(k) & \sim k^{z^{*}-2+\tilde{\alpha} / \nu} .
\end{aligned}
$$

Ces quantités sont dans tous les cas non divergentes, ce qu'on peut assigner au fait qu'elles sont déterminées par le libre parcours moyen, lequel ne dépend pour ces systèmes que du comportement à courte distance (grands vecteurs d'ondes). De nombreux systèmes physiques (ferromagnétiques et antiferromagnétiques isotropes, hélium, etc...) possèdent des coefficients de transport divergents pour $k \rightarrow 0$ et des couplages de type mode-mode ont été introduits $[13,14]$ qui peuvent conduire à des forces effectives à longue portée invalidant l'argument naïf précédent et à des coefficients de transport divergents.

4. Modèles avec couplage mode-mode. - Ce type de modèle est clairement présenté et, est analysé en détail pour le système de Heisenberg isotrope, par Ma et Mazenko [3].

4.1 Terme DE COUPlage MOde-Mode [3]. - Soit $\psi_{j}$ l'ensemble des variables pertinentes (paramètre d'ordre et variables conservées). Les termes de couplage mode-mode introduits par Kawasaki [13] et Kadanoff-Swift [14] peuvent s'écrire [15]

$V_{i}[\psi]=v_{0} \sum_{i}\left[\frac{\delta}{\delta \psi_{j}} Q_{i j}[\psi]-Q_{i j}[\psi] \frac{\delta \mathcal{H e}\{\psi\}}{\delta \psi_{j}}\right]$

où $Q_{i j}$ est construit à partir des crochets de Poisson ou commutateurs des $\psi_{j}$, d'où il suit que $Q_{i j}=-Q_{j i}$. En gros, on peut dire que si l'ensemble des paramètres d'ordre $(\varphi)$ possède un groupe de symétrie, il faudra garder les commutateurs des générateurs de ce groupe avec les $\varphi$, ces générateurs étant eux-mêmes nécessairement des variables conservées. Dans le cas du système de Heisenberg isotrope, les générateurs et les $\varphi$ sont confondus et

$$
Q_{i j}=\left[\varphi_{i}, \varphi_{j}\right]=\varepsilon_{i j k} \varphi_{k}
$$


On notera que l'identité

$$
\sum_{j} \frac{\delta}{\delta \psi_{j}} V_{j}[\psi] \mathrm{e}^{-\mathscr{H}\{\psi\}}=0
$$

qui découle de (51) et exprime la conservation du courant de probabilité dans l'espace des $\psi$, entraîne que le terme $V_{j}[\psi]$ ne change pas la probabilité d'équilibre $\exp \{-\mathfrak{H}\}$.

L'équation stochastique générale gouvernant des systèmes avec couplage mode-mode a donc la forme :

$$
\begin{array}{r}
\frac{\partial}{\partial t} \psi_{j}(t)=-\Gamma_{j}\left[\frac{\partial \mathcal{J}\{\{\}}{\partial \psi_{j}(t)}+h_{j}(t)\right]+V_{j}[\psi]- \\
-v_{0} \sum_{l} Q_{j l}[\psi] h_{l}+\eta_{j}(t) .
\end{array}
$$

4.2 Lagrangien de Martin, Siggia, Rose [16]. Il n'existe pas de Lagrangien simple du type (18) associé à (54). Si on veut construire un Lagrangien afin d'utiliser les méthodes générales ci-dessus on doit avoir recours à l'artifice introduit par Martin, Siggia et Rose [16] qui peut se formuler de la façon suivante. Ecrivons (54) sous la forme

$$
K_{j}\{\psi\}-\Gamma_{j}^{-1} \eta_{j}(t)=0
$$

où $K_{j}$ est la fonctionnelle de $\psi$ définie par (54) et introduisons une variable conjuguée $\hat{\psi}_{j}$ associée à chaque $\psi_{j}$. La fonction génératrice des fonctions de corrélation des $\psi_{j}$

$$
\begin{aligned}
Z_{\eta}(l)=\int \mathcal{D} \psi \mathcal{D} \hat{\psi} & \exp \times \\
& \times\left\{-\int \mathrm{d}^{d} x \mathrm{~d} t \sum_{j}\left[\hat{\psi}_{j}(t) K_{j}\{\psi\}\right.\right. \\
& \left.\left.-\Gamma_{j}^{-1} \hat{\psi}_{j}(t) \eta_{j}(t)+l_{j}(t) \psi_{j}(t)\right]\right\}
\end{aligned}
$$

restitue (55) par intégration sur $\hat{i} \hat{\psi}$. En prenant la moyenne sur les $\eta_{j}$ de $Z_{\eta}(l) / Z_{\eta}(0)$, compte tenu du fait que $Z_{\eta}(0)=1$, on obtient :

$$
\begin{aligned}
Z(l)=<Z_{\eta}(l)> & =\int \mathscr{D} \boldsymbol{D} \hat{\psi} \exp \times \\
& \times\left\{-\int \mathrm{d}^{d} x \mathrm{~d} t \sum_{j}\left[\hat{\psi}_{j}(t) K_{j}\{\psi\}\right.\right. \\
& \left.\left.+\Gamma_{j}^{-1} \hat{\psi}_{j}(t) \hat{\psi}_{j}(t)+l_{j}(t) \psi_{j}(t)\right]\right\}
\end{aligned}
$$

définissant le Lagrangien de MSR

$$
Z(l)=\int \mathscr{D} \psi \mathfrak{D} \hat{\psi} \exp \left\{+\mathfrak{L}_{\mathrm{MSR}}\right\}
$$

Naturellement le prix à payer est que l'on a deux fois plus de fonctions de corrélations élémentaires, outre $\langle\psi \psi\rangle$ on a aussi $\langle\widehat{\psi} \psi\rangle$ (l'absence de termes en $\psi \psi$ dans $\mathcal{L}_{\mathrm{MSR}}$ entraîne $\langle\widehat{\psi} \hat{\psi}\rangle=0$ ). Ces deux types de fonction de corrélation sont reliés par une relation de fluctuation-dissipation [3, 17]. Dans la limite où le terme mode-mode disparaît on peut montrer [17] que $\mathcal{L}_{\text {MSR }}$ redonne la formulation (18) où un seul type de fonction de corrélation apparaît. Il faut cependant tenir compte du fait qu'en général les limites à fréquence nulle des fonctions de corrélation ne s'identifient pas avec les limites statiques. Enfin, il faut tenir compte de la loi de conservation (53) qui, via une identité de Ward, gouverne la façon dont se renormalise la constante de couplage [2], $v_{0}$ et détermine la fonction de Wilson supplémentaire $W_{v}$ associée à ce nouveau couplage. La discussion des points fixes et de leur stabilité, des exposants critiques et des corrections principales suit alors le même cours que dans les modèles discutés plus haut.

\section{Bibliographie}

[1] Halperin, B. I., Hohenberg, P. C., Ma, S. K., Phys. Rev. Lett. 29 (1972) 1548 ; Phys. Rev. B 10 (1974) 139.

[2] Halperin, B. I., Hohenberg, P. C., Siggia, E., Phys. Rev. Lett. 32 (1974) 1289, preprint 1975.

[3] MA, S. K., Mazenko, E., Phys. Rev. B 11 (1975) 4077.

[4] Wilson, K., Kogut, J., Phys. Rep. C 12 (1974) 75.

[5] Brézin, E., Le GuIllou, J. C., ZinN-Justin, J., Field Theoretical Approach to Critical Phenomena (édité par C. Domb et M. Green) Vol. VI (1975) et preprint Saclay DPh-T/74/100.

[6] Ferrell, R., Menyhard, N., Schmidt, H., Schwabl, F., Szepfalusy, P., Phys. Rev. Lett. 18 (1967) 891.

[7] Halperin, B., Hohenterg, P. C., Phys. Rev. 177 (1969) 952.

[8] De Dominicis, C., Brézin, E., ZinN-Justin, J., preprint Saclay DPh-T/75/17.
[9] BRÉzin, E., De Dominicis, C., preprint Saclay DPh-T/75/58. [10] Glauber, R., J. Math. Phys. 4 (1963) 294.

[11] De Dominicis, C., Lett. Nuovo Cimento 12 (1975) 567.

[12] Yahata, H., J. Phys. Soc. Japan 30 (1971) 657.

[13] Kadanoff, L., Swift, J., Ann. Phys. 50 (1968) 312.

[14] Kawasaki, K., Ann. Phys. 61 (1970) 1.

[15] Mori, A., Fujisaka, H., Progr. Theor. Phys. 49 (1973) 764

Mori, A., Fujisaka, H., Shigematsu, H., Progr. Theor. Phys. 51 (1974) 109.

[16] Martin, P. C., Siggia, E., Rose, H., Phys. Rev. A 8 (1973) 423.

[17] Deker, U., HAAKe, F., Essen preprint 1975. 